J. N. Pandey and R. S. Pathak

Nagoya Math. J.

Vol. 72 (1978), 1-25

\title{
EIGENFUNCTION EXPANSION OF GENERALIZED FUNCTIONS
}

\author{
J. N. PANDEY AND R. S. PATHAK
}

\section{Introduction}

Expansions of generalized functions have been investigated by many authors. Korevaar [11], Widlund [20], Giertz [8], Walter [19] developed procedures for expanding generalized functions of Korevaar [12], Temple [17], and Lighthill [13]. Expansions of certain Schwartz distributions [15] into series of orthonormal functions were given by Zemanian [23] (see also Zemanian [24]) and thereby he extended a number of integral transforms to distributions. The method involved in his work is very much related to the Hilbert space technique and is of somewhat different character from those used in most of the works on integral transforms such as [24, chapters 1-8]. Other works that discuss orthogonal series expansions involving generalized functions are by Bouix [1, chapter 7], Braga and Schönberg [2], Gelfand and Shilov [7, vol. 3, chapter 4] and Warmbrod [21].

In this paper, expansions of generalized functions (distributions) with respect to some general classes of complete orthonormal systems are investigated. For this, a testing function space $\mathscr{N}(I)$ is constructed over the closed interval $I=[a, b]$ containing the normalized eigen functions $\left\{\Psi_{n}(x)\right\}_{n=1}^{\infty}$ of the Sturm-Liouville system. The Sturm-Liouville transform $F(n)$ of a generalized function $f \in \mathscr{N}^{\prime}(I)$ is defined by

$$
F(n)=\left\langle f(x), \Psi_{n}(x)\right\rangle \quad n=1,2,3, \cdots
$$

It is shown in section 4 that

$$
\lim _{N \rightarrow \infty}\left\langle\sum_{n=1}^{N} F(n) \Psi_{n}(x), \varphi(x)\right\rangle=\langle f, \varphi\rangle, \quad \forall \varphi \in D(I)
$$

where the above convergence is interpreted in the weak distributional

Received June 25, 1976. 
sense. In section 5 we have discussed special cases of interest and in sections 8 and 9 we have utilized our results in solving certain differential equations with distributional boundary conditions. The notation and terminology of this work follow those of [18], [22] and [24].

\section{A general expansion theorem}

The following theorem due to Yosida [22, p. 173] plays a fundamental role in our present investigation.

THEOREM 1 (Yosida). Let $q(x)$ be a real-valued continuous function in a finite or infinite closed interval $[a, b]$. We make no assumption concerning the behaviour of $q(x)$ at the boundary points; as $x \rightarrow a$, or as $x \rightarrow b, q(x)$ may tend to finite limits, may tend to $\pm \infty$, may have no limits. Let $f(x)$ be a real-valued function satisfying the conditions

(i) $f^{\prime \prime}(x)$ is continuous on $(-\infty, \infty)$.

(ii) For some $a^{\prime}, b^{\prime}, a<a^{\prime}<b^{\prime}<b$,

$$
f(x) \equiv 0 \text { on semi-infinite intervals }-\infty<x \leq a^{\prime}, b^{\prime} \leq x<\infty .
$$

Let $\left\{\lambda_{n, a, b}\right\}$ be the set of all eigenvalues of the boundary value problem

$$
\left\{\begin{array}{l}
L_{x} \phi=\lambda \phi, \quad L_{x} \equiv q(x)-\frac{d^{2}}{d x^{2}} \\
\phi(a) \cos \alpha+\phi^{\prime}(a) \sin \alpha=0 \\
\phi(b) \cos \beta+\phi^{\prime}(b) \sin \beta=0
\end{array}\right.
$$

and $\left\{\phi_{n, a, b}\right\}$ be the corresponding orthonormal system of the eigenfunctions. Then $f(x)$ can be expanded into the Fourier series

$$
\begin{gathered}
f(x)=\sum_{n=1}^{\infty} f_{n, a, b} \phi_{n, a, b}(x) \\
f_{n, a, b}=\left(f, \phi_{n, a, b}\right)=\int_{a}^{b} f(x) \overline{\phi_{n, a, b}}(x) d x .
\end{gathered}
$$

Without any loss of generality we can assume that the eigenfunctions of the system (2.1) are real. (2.2) converges absolutely and uniformly on the interval $[a, b]$.

Remark. The eigenfunction expansion as given in Theorem 1 is also valid when $f(x)$ is a complex-valued function belonging to $C_{2}(-\infty, \infty)$ 
and having support contained in the open interval $\left(a^{\prime}, b^{\prime}\right)$ where $a<a^{\prime}$ $<b^{\prime}<b$. The proof can be given by using Theorem 1 for the real and imaginary parts of $f$ separately.

COROLLARY 1. Let $f(x) \in C_{2}[a, b]$ with support contained in the open interval $(a, b)$. Then the Fourier series expansion

$$
\begin{gathered}
\frac{1}{(b-a)} \int_{a}^{b} f(y) d y+\frac{2}{(b-a)} \sum_{n=1}^{\infty} \cos \left(\frac{n \pi(x-a)}{(b-a)}\right) . \\
\int_{a}^{b} f(y) \cos \left(\frac{n \pi(y-a)}{(b-a)}\right) d y
\end{gathered}
$$

of $f(x)$ converges absolutely and uniformly to $f(x)$ over the interval $[a, b]$.

Proof. The normalized eigenfunctions of the Sturm-Liouville system

$$
\left\{\begin{array}{l}
\frac{d^{2} y}{d x^{2}}+\lambda y=0 \quad a \leq x \leq b \\
y^{\prime}(a)=0 \\
y^{\prime}(b)=0
\end{array}\right.
$$

are $\frac{1}{\sqrt{b-a}}, \sqrt{\frac{2}{b-a}} \cos \left(\frac{n \pi(x-a)}{(b-a)}\right), n=1,2,3, \ldots$. The result now follows in view of Theorem 1 .

COROLLARY 2. Let $f(x) \in C_{2}[0, \pi]$ with support contained in the open interval $(0, \pi)$. Then the Fourier series expansion

$$
\frac{1}{\pi} \int_{0}^{\pi} f(y) d y+\frac{2}{\pi} \sum_{n=1}^{\infty} \cos n x \int_{0}^{\pi} f(y) \cos n y d y
$$

of $f(x)$ converges absolutely and uniformly to $f(x)$ over the interval $[0, \pi]$.

Proof. This is an immediate consequence of Corollary 1 with $a=0$ and $b=1$. The normalized eigenfunctions of the system

$$
\left\{\begin{array}{l}
\frac{d^{2} y}{d x^{2}}+\lambda y=0 \quad 0 \leq x \leq \pi \\
y^{\prime}(0)=0 \\
y^{\prime}(\pi)=0
\end{array}\right.
$$

turn out to be 


$$
\Psi_{0}(x)=\frac{1}{\sqrt{\pi}}, \quad \Psi_{n}(x)=\sqrt{\left(\frac{2}{\pi}\right)} \cos n x, \quad n=1,2,3, \ldots
$$

Unless otherwise stated for the sake of convenience we shall write $\Psi_{n}(x)$ for $\varphi_{n, a, b}(x)$ and $\lambda_{n}$ for $\lambda_{n, a, b}$.

\section{The testing function space $\mathscr{N}(I)$}

Let $I$ denote the open interval $(a, b)$ where $a$ and $b$ are finite such that $-\infty<a<b<\infty$ and let $x$ and $t$ be real variables restricted to $I$. Then the space $\mathscr{N}(I)$ consists of all the complex valued infinitely differentiable functions $\varphi(x)$ defined over $I$ such that

$$
\gamma_{k}(\varphi) \triangleq \sup _{a<x<b}\left|\Delta_{x}^{k} \varphi(x)\right|<\infty,
$$

for all $k=0,1,2, \cdots$, where the operator $\Delta_{x}$ is defined by

$$
\Delta_{x}=\frac{d^{2}}{d x^{2}}-q(x)=-L_{x}
$$

$q(x)$ being assumed to be infinitely differentiable in $(a, b)$. The topology on $\mathscr{N}(I)$ is defined by the separating collection of seminorm $\left\{\gamma_{k}\right\}_{k=0}^{\infty}[24$, pp. 7-10]. A sequence $\left\{\varphi_{\nu}\right\}_{\nu=1}^{\infty}$ is said to converge in $\mathscr{N}(I)$ to the limit $\varphi$ if $\gamma_{k}\left(\varphi_{\nu}-\varphi\right) \rightarrow 0$ as $\nu \rightarrow \infty$ for each $k=0,1,2, \ldots$ A sequence $\left\{\varphi_{\nu}\right\}_{\nu=1}^{\infty}$ is said to be a Cauchy sequence in $\mathscr{N}(I)$ if $\gamma_{k}\left(\varphi_{\nu}-\varphi_{\mu}\right)$ tends to zero as $\nu$ and $\mu$ both tend to infinity independently of each other. It can be readily seen that $\mathscr{N}(I)$ is a locally convex, sequentially complete [24, pp. 176-177] Hausdorff topological vector space. The dual of $\mathscr{N}(I)$ will be represented by $\mathscr{N}^{\prime}(I)$. The space $D(I)$ is a subspace of $\mathscr{N}(I)$ and the restriction of $f \in D^{\prime}(I)$ to $\mathscr{N}(I)$ is in $D^{\prime}(I)$.

LEMMA 1. Let $\phi(x) \in D(I), I=(a, b)$ and let $\left\{\Psi_{n}(x)\right\}_{n=1}^{\infty}$ be the normalized eigenfunction of the system (2.1).

Define $S_{N}(t, x)=\sum_{n=1}^{N} \Psi_{n}(x) \Psi_{n}(t)$.

Then

$$
\int_{a}^{b} S_{N}(t, x) \phi(x) d x \rightarrow \phi(t) \quad \text { in } \mathscr{N}(I) \text { as } N \rightarrow \infty
$$

Proof. A simple computation shows that 


$$
\begin{aligned}
\Delta_{t}^{k} \int_{a}^{b} S_{N}(t, x) \phi(x) d x & =\int_{a}^{b} S_{N}(t, x) \Delta_{x}^{k} \phi(x) d x \\
& =\int_{a}^{b} S_{N}(t, x) \phi_{k}(x) d x
\end{aligned}
$$

where $\phi_{k}(x)=\Delta_{x}^{k} \phi(x)$.

Now

$$
\Delta_{t}^{k}\left[\int_{a}^{b} S_{N}(t, x) \phi(x) d x-\phi(t)\right]=\int_{a}^{b} S_{N}(t, x) \phi_{k}(x) d x-\phi_{k}(t)
$$

which tends to zero as $N \rightarrow \infty$ uniformly for all $t \in[a, b]$ by Theorem 1 and the remark following it.

4. The Sturm-Liouville expansion of generalized functions of $\mathscr{N}^{\prime}(I): I=(a, b)$

We will now prove the following inversion theorem for the distributional finite Sturm-Liouville transform which gives rise to an expansion of $f \in \mathscr{N}^{\prime}(I), I=(a, b)$ where $a$ and $b$ are finite such that $-\infty<a$ $<b<\infty$.

THEOREM 2. Let $f$ be an arbitrary element of $\mathscr{N}^{\prime}(I)$ and let $\Psi_{n}(x)$ be the normalized eigenfunctions of the Sturm-Liouville problem (2.1) which are assumed to be elements of $\mathscr{N}(I)$. Define the distributional finite Sturm-Liouville transform of $f$ by

$$
S[f] \triangleq F(n) \triangleq\left\langle f(x), \Psi_{n}(x)\right\rangle,
$$

for each $n=1,2,3, \cdots$ then for each $\varphi(t) \in D(I)$,

$$
\lim _{N \rightarrow \infty}\left\langle\sum_{n=1}^{N} \Psi_{n}(t) F(n), \varphi(t)\right\rangle=\langle f(t), \varphi(t)\rangle
$$

Proof. Let us assume that the support of $\varphi(x)$ is contained in the finite interval $(c, d)$ where $a<c<d<b$. Our theorem will be proved by justifying the steps in the following manipulations.

$$
\begin{aligned}
\left\langle\sum_{n=1}^{N} \Psi_{n}(t)\right. & F(n), \varphi(t)\rangle \\
= & \int_{c}^{a} \sum_{n=1}^{N} \Psi_{n}(t) F(n) \varphi(t) d t \\
= & \int_{a}^{b} \sum_{n=1}^{N}\left\langle f(x), \Psi_{n}(x)\right\rangle \Psi_{n}(t) \varphi(t) d t
\end{aligned}
$$




$$
\begin{aligned}
& =\int_{a}^{b}\left\langle f(x), \sum_{n=1}^{N} \Psi_{n}(x) \Psi_{n}(t)\right\rangle \varphi(t) d t \\
& =\left\langle f(x), \int_{a}^{b} \sum_{n=1}^{N} \Psi_{n}(x) \Psi_{n}(t) \varphi(t) d t\right\rangle \\
& =\left\langle f(x), \int_{a}^{b} S_{N}(t, x) \varphi(t) d t\right\rangle \\
& \rightarrow\langle f(x), \varphi(x)\rangle \quad \text { as } N \rightarrow \infty .
\end{aligned}
$$

The step (4.3) is justified by using the fact that the function $\sum_{n=1}^{N} \Psi_{n}(t) F(n)$ is locally integrable over the interval $(a, b)$, and $\varphi(x)$ $\in D(I)$. The step (4.5) follows by the linearity of the functional. Equality of the expressions (4.5) and (4.6) follows by the linearity property of generalized functions. That the expression (4.7) goes to (4.8) as $N \rightarrow \infty$ follows from Lemma 1. This completes the proof of the theorem.

THEOREM 3 (Uniqueness). Let $f, g \in \mathscr{N}^{\prime}(I), I=(a, b)$ where $-\infty<a$ $<b<\infty$ and let us define $S[f]=F(n)$ and $S[g]=G(n)$ for all $n=1,2$, $\cdots$ If $F(n)=G(n)$ for all $n=1,2,3, \cdots$, then $f=g$ in the sense of equality over $D(I)$.

The proof is obvious in view of the above inversion theorem.

\section{Special cases}

In the following lines we list a number of particular values of $q(x)$, corresponding eigenfunctions $\Psi_{n}$ and eigenvalues $\lambda_{n}$, and the intervals $I$ on which expansion is applicable. Appropriate generalized functions and their expansions are also given.

\section{Expansion in Fourier series}

$$
I=(-\pi, \pi), \quad q(x)=0 .
$$

The Sturm Liouville-problem is described by

$$
\frac{d^{2} y}{d x^{2}}+\lambda y=0 ; \quad y(-\pi)=y(\pi) \quad \text { and } \quad y^{\prime}(-\pi)=y^{\prime}(\pi)
$$

For this problem 


$$
\Psi_{n}(x)=\left\{\begin{array}{cc}
\frac{1}{\sqrt{(2 \pi)}} & \text { for } n=0 \\
\frac{\cos n x}{\sqrt{\pi}} & \text { for } n=2 k \\
\frac{\sin n x}{\sqrt{\pi}} & \text { for } n=2 k-1 \\
\lambda_{n}=n^{2} . & (k=1,2,3, \cdots)
\end{array}\right.
$$

In the present case the testing function space $\mathscr{F}(I), l=(-\pi, \pi)$ consists of complex-valued $C^{\infty}$ functions $\phi(x)$ defined over $I$ satisfying the conditions

$$
\gamma_{k}(\phi)=\sup _{-\pi<x<\pi}\left|D^{2 k} \phi(x)\right|<\infty, \quad k=0,1,2, \cdots .
$$

The topology over $\mathscr{F}(I)$ is generated by the sequence of seminorms $\left\{\gamma_{k}\right\}_{k=0}^{\infty}[24$, p. 8] and the concept of convergence and completeness over $\mathscr{F}(I)$ is defined in the usual way. $\mathscr{F}^{\prime}(I)$ denotes the dual of $\mathscr{F}(I)$.

Now, the Fourier expansion of any $f \in \mathscr{F}^{\prime}(I)$ can be stated by

THEOREM 4. Let $f \in \mathscr{F}^{\prime}(I), I=(-\pi, \pi)$ and $F(n)$ be the Fourier transform of $f$ for each $n=0,1,2, \cdots$, defined by

$$
F(n)=\left\langle f(x), \Psi_{n}(x)\right\rangle
$$

where $\Psi_{n}(x)$ are defined by (5.3). Then for each $\varphi(x) \in D(I)$,

$$
\lim _{N \rightarrow \infty}\left\langle\sum_{n=0}^{N} \Psi_{n}(x) F(n), \varphi(x)\right\rangle=\langle f(x), \varphi(x)\rangle .
$$

\section{Expansion in a series of Jacobi polynomials}

$$
\begin{aligned}
& I=(-\pi / 2, \pi / 2) \text { and } \\
& q(x)=-\frac{1}{4} \tan ^{2} x-\frac{1}{2}+\frac{1}{2}(\alpha+\beta) \\
& -\frac{1}{4} \sec ^{2} x(\beta-\alpha-(\alpha+\beta) \sin x)(\beta-\alpha-(\alpha+\beta) \sin x \\
& +2 \sin x), \quad \alpha>-1, \beta>-1 \text {. }
\end{aligned}
$$

The Sturm-Liouville problem is

$$
\frac{d^{2} y}{d x^{2}}+(\lambda-q(x)) y=0,
$$

$y(-\pi / 2)$ and $y(\pi / 2)$ are finite.

In this case 


$$
\Psi_{n}(x)=\left(\frac{\cos x w(\sin x)}{h_{n}}\right)^{1 / 2} P_{n}^{(\alpha, \beta)}(\sin x), \quad n=0,1,2, \ldots
$$

where

$$
\begin{gathered}
w(x)=(1-x)^{\alpha}(1+x)^{\beta} \\
h_{n}=2^{\alpha+\beta+1} \frac{\Gamma(n+\alpha+1) \Gamma(n+\beta+1)}{n !(2 n+\alpha+\beta+1) \Gamma(n+\alpha+\beta+1)}
\end{gathered}
$$

and $P_{n}^{(\alpha, \beta)}(x)$ is the Jacobi polynomial of degree $n$ [5].

$$
\lambda_{n}=n(n+\alpha+\beta+1) .
$$

The testing function space $\mathscr{J}^{(\alpha, \beta)}(I), I=(-\pi / 2, \pi / 2)$ in the present case consists of infinitely differentiable complex valued functions $\varphi(x)$ defined over $I$ such that

$$
\begin{array}{r}
\gamma_{k}(\varphi)=\sup _{-\pi / 2<x<\pi / 2}\left|\left(D^{2}-q(x)\right)^{k} \varphi(x)\right|<\infty, \\
\text { for each } k=0,1,2, \cdots
\end{array}
$$

where $q(x)$ is given by (5.7). The topology over $\mathscr{J}^{(\alpha, \beta)}$ is generated by the collection of seminorms $\left\{\gamma_{k}\right\}_{k=0}^{\infty}\left[24\right.$, p. 8]. Notice that $\Psi_{n}(x)$ $=\left(\frac{\cos x w(\sin x)}{h_{n}}\right)^{1 / 2} P^{(\alpha, \beta)}(\sin x) \in \mathscr{J}_{n}^{(\alpha, \beta)}(I)$ for $\alpha, \beta \geq-\frac{1}{2}$ and $n=0,1,2, \cdots$. The dual space of $\mathscr{J}^{(\alpha, \beta)}$ is denoted by $\mathscr{J}^{(\alpha, \beta)^{\prime}}$. The corresponding expansion formula is described by

THEOREM 5. Let $f \in \mathscr{J}^{(\alpha, \beta)^{\prime}}(I), I=(-\pi / 2, \pi / 2), \alpha, \beta \geq-\frac{1}{2}$, and let $F(n)$ be the distributional Jacobi transform of $f$ defined by

$$
F(n)=\left\langle f(x), \Psi_{n}(x)\right\rangle
$$

where $\Psi_{n}(x)$ is given by (5.9). Then, for each $\varphi \in D(I)$.

$$
\lim _{N \rightarrow \infty}\left\langle\sum_{n=0}^{N} \Psi_{n}(x) F(n), \varphi(x)\right\rangle=\langle f(x), \varphi(x)\rangle
$$

3. Expansion in a series of Legendre polynomials over $(-\pi / 2, \pi / 2)$

$$
I=(-\pi / 2, \pi / 2), \quad q(x)=-\frac{1}{4} \tan ^{2} x-\frac{1}{2} .
$$

The corresponding Sturm-Liouville problem is

$$
\frac{d^{2} y}{d x^{2}}+\left(\lambda+\frac{1}{4} \tan ^{2} x+\frac{1}{2}\right) y=0,
$$


where $y(-\pi / 2)$ and $y(\pi / 2)$ are finite. In this case the eigenfunctions become

$$
\Psi_{n}(x)=\left(\left(n+\frac{1}{2}\right) \cos x\right)^{1 / 2} P_{n}(\sin x), \quad n=0,1,2, \cdots
$$

where $P_{n}(x)$ is the Legendre polynomial of degree $n$.

$$
\lambda_{n}=n(n+1) \text {. }
$$

This is a special case of the Jacobi series expansion and is obtained on setting $\alpha=\beta=0$. Therefore the expansion formula is given by

THEOREM 6. Let $f \in \mathscr{J}^{(0,0)^{\prime}}(I), I=(-\pi / 2, \pi / 2)$ and let $F(n)$ be the distributional Legendre transform of $f$ defined by

$$
F(n)=\left\langle f(x),\left(\left(n+\frac{1}{2}\right) \cos x\right)^{1 / 2} P_{n}(\sin x)\right\rangle .
$$

Then for each $\varphi(t) \in D(I)$

$$
\lim _{N \rightarrow \infty}\left\langle\sum_{n=0}^{N}\left(\left(n+\frac{1}{2}\right) \cos x\right)^{1 / 2} P_{n}(\sin x) F(n), \varphi(x)\right\rangle=\langle f(x), \varphi(x)\rangle .
$$

The expansions in Chebyshev polynomials [5] and in Gegenbauer polynomials [5] are obtained merely on setting $\alpha=\beta=-\frac{1}{2}$ and $\alpha=\beta$ $=\rho-\frac{1}{2}$ in the Jacobi polynomial case respectively.

Now we wish to change the range of definition of the Legendre transform from $(-\pi / 2, \pi / 2)$ to $(-1,1)$. Applying the change of variable $x=\sin ^{-1} t$, we have

$$
\frac{d^{2}}{d x^{2}}+\frac{1}{4} \tan ^{2} x+\frac{1}{2}=\left(1-t^{2}\right) \frac{d^{2}}{d t^{2}}-t \frac{d}{d t}+\frac{2-t^{2}}{4\left(1-t^{2}\right)}=\nabla_{t} \quad \text { (say) . }
$$

If $f \in \mathscr{J}^{(0,0)}(I), I=(-\pi / 2, \pi / 2), \phi(x) \in \mathscr{J}^{(0,0)}(I)$, then we write

$$
\begin{aligned}
\langle f(x), \phi(x)\rangle & =\left\langle f\left(\sin ^{-1} t\right),\left(1-t^{2}\right)^{-1 / 2} \phi\left(\sin ^{-1} t\right)\right\rangle \\
& =\langle\hat{f}(t), \Psi(t)\rangle,
\end{aligned}
$$

where $\hat{f}(t)=f\left(\sin ^{-1} t\right)$ and $\Psi(t)=\left(1-t^{2}\right)^{-1 / 2} \phi\left(\sin ^{-1} t\right)$, Also, from the definition of $\mathscr{J}^{(0,0)}(I)$, we have

$$
\begin{aligned}
\gamma_{k}(\varphi) & =\sup _{-\pi / 2<x<\pi / 2}\left|\left(\frac{d^{2}}{d x^{2}}+\frac{1}{4} \tan ^{2} x+\frac{1}{2}\right)^{k} \phi(x)\right| \\
& =\sup _{-1<t<1}\left|\nabla_{t}^{k}\left(\left(1-t^{2}\right)^{1 / 2} \Psi(t)\right)\right| \\
& =\rho_{k}(\Psi) \quad \text { (say). }
\end{aligned}
$$


It is easily seen that the mapping $\phi(x) \not \rightarrow\left(1-t^{2}\right)^{1 / 2} \Psi(t)$ is an isomorphism from $\mathscr{J}^{(0,0)}$ to $\mathscr{P}(I), I=(-1,1)$, where $\mathscr{P}(I)$ stands for the testing function space which consists of all complex valued and infinitely differentiable functions $\Psi(t)$ satisfying (5.19). The topology over $\mathscr{P}(I)$ is generated by the sequence of seminorms $\left\{\rho_{k}\right\}_{k=0}^{\infty}$. The concept of convergence and completeness in $\mathscr{P}(I)$ is defined in the usual way. Consequently, $\hat{f}(t) \in \mathscr{P}^{\prime}(I)$. As a result, the inversion formula for the Legendre transformation given by Theorem 6 , will be applicable to generalized functions in the space $\mathscr{P}^{\prime}(I)$. Thus we arrive at

THEOREM 7. Let $f \in \mathscr{P}^{\prime}(I), I=(-1,1)$ and let $F(n)$ be the distributional Legendre transform of $f$ defined by

$$
F(n)=\left\langle f(t), P_{n}(t)\right\rangle .
$$

Then for each $\varphi(t) \in D(I)$,

$$
\lim _{N \rightarrow \infty}\left\langle\sum_{n=0}^{N}\left(n+\frac{1}{2}\right) P_{n}(t) F(n), \varphi(t)\right\rangle=\langle f(t), \varphi(t)\rangle .
$$

\section{Expansion in series of Bessel functions}

These expansions can be classified in three different forms depending upon the interval $I$ and the boundary conditions involved in the Sturm-Liouville problem.

First form:

$$
I=(0,1) ; q(x)=\frac{\nu^{2}-\frac{1}{4}}{x^{2}}, \quad \nu \geq-\frac{1}{2}
$$

The Sturm-Liouville problem can be described by

$$
\frac{d^{2} y}{d x^{2}}+\left(\lambda-\frac{\nu^{2}-\frac{1}{4}}{x^{2}}\right) y=0, \quad y(0)=0, y(1)=0 \text {. }
$$

Here

$$
\Psi_{n}(x)=\sqrt{2 x} J_{\nu}\left(\xi_{n} x\right) / J_{\nu+1}\left(\xi_{n}\right), \quad n=1,2,3, \cdots
$$

where $J_{\nu}(x)$ is the 2 th order Bessel function of the first kind and $\xi_{n}$ denote all the positive roots of $J_{\nu}(\xi)=0,0<\xi_{1}<\xi_{2}<\ldots$.

$$
\lambda_{n}=\xi_{n}^{2} \text {. }
$$


In this case the expansion is called the $\nu$ th order Fourier-Bessel series for $f$. For the present problem we define the testing function space $\mathscr{H}_{\nu}(I), I=(0,1)$ to be the collection of all infinitely differentiable complex valued functions $\varphi(x)$ satisfying

$$
\gamma_{k}(\varphi)=\sup _{0<x<1}\left|\left(\frac{d^{2}}{d x^{2}}-\frac{\nu^{2}-\frac{1}{4}}{x^{2}}\right)^{k} \varphi(x)\right|<\infty,
$$

for each $k=0,1,2, \cdots$. The topology over $\mathscr{H}_{\nu}(I)$ is generated by the separating collection of seminorms $\left\{\gamma_{k}\right\}_{k=0}^{\infty}$. The concept of convergence and completeness is defined in the usual way. The corresponding dual space is denoted by $\mathscr{H}_{\nu}^{\prime}(I)$.

Using Theorem 2 we now have the following result.

THEOREM 8. Let $f \in \mathscr{H}_{\nu}^{\prime}(I), I=(0,1)$ where $\nu \geq-\frac{1}{2}$, and let $F\left(\xi_{n}\right)$ be the finite Hankel transform of $f$ for each $n=1,2,3, \cdots$, defined by

$$
F\left(\xi_{n}\right)=\left\langle f(x), \sqrt{x} J_{\nu}\left(\xi_{n} x\right)\right\rangle .
$$

Then for each $\varphi(x) \in D(I)$, we have

$$
\lim _{N \rightarrow \infty}\left\langle 2 \sqrt{x} \sum_{n=1}^{N} \frac{F\left(\xi_{n}\right)}{J_{\nu+1}^{2}\left(\xi_{n}\right)} J_{\nu}\left(\xi_{n} x\right), \varphi(x)\right\rangle=\langle f(x), \varphi(x)\rangle .
$$

Remark. A similar expansion formula has been obtained after an elaborate analysis by Dube [4]. The result proved by Dube is the best possible for $\alpha=\frac{1}{2}$. Now using the fact that

$$
\Delta_{x}^{k} \varphi(x)=x^{1 / 2} \Omega_{x}^{k}\left(\frac{\varphi(x)}{\sqrt{x}}\right)
$$

where

$$
\Delta_{x}=\frac{d^{2}}{d x^{2}}-\frac{\nu^{2}-\frac{1}{4}}{x^{2}}
$$

and

$$
\Omega_{x}=\frac{d^{2}}{d x^{2}}+\frac{1}{x} \frac{d}{d x}-\frac{\nu^{2}}{x^{2}}
$$

and using transformation analogous to that used in [24, p. 245] the result of Dube can be derived from Theorem 8 . 
Second form:

$$
I=(0,1) ; q(x)=\frac{\nu^{2}-\frac{1}{4}}{x^{2}}, \quad \nu \geq-\frac{1}{2} .
$$

The Sturm-Liouville problem is

$$
\frac{d^{2} y}{d x^{2}}+\left(\lambda-\frac{\nu^{2}-\frac{1}{4}}{x^{2}}\right) y=0 ; y(0)=0, \quad y^{1}(1)+H y(1)=0
$$

where $H$ is any fixed real number. In this case

$$
\Psi_{n}(x)=\sqrt{\frac{2 x}{h_{n}}} J_{\nu}\left(\xi_{n} x\right) \quad n=1,2,3, \cdots
$$

where $\xi_{n}$ denote all the positive roots of

$$
\xi J_{\nu}^{(1)}(\xi)+H J_{\nu}(\xi)=0
$$

with $0<\xi_{1}<\xi_{2}<\ldots$ and $J_{\nu}^{(1)}(\xi)=\frac{d}{d \xi} J_{\nu}(\xi)$. Also

$$
h_{n}=\left[J_{\nu}^{(1)}\left(\xi_{n}\right)\right]^{2}+\left(1-\nu^{2} \xi_{n}^{-2}\right)\left[J_{\nu}\left(\xi_{n}\right)\right]^{2} .
$$

$$
\lambda_{n}=\xi_{n}^{2} \text {. }
$$

The expansion of $f$ is called Dini series for $f$ which is given by the following theorem.

THEOREM 9. Let $f \in \mathscr{H}_{\nu}^{\prime}(I)$ where $\nu \geq-\frac{1}{2}$, and let $F\left(\xi_{n}\right)$ be the finite Hankel transform of the second form of $f$, where $\xi_{n}$ are the positive roots of (5.33), defined by

$$
F\left(\xi_{n}\right)=\left\langle f(x), \sqrt{x} J_{\nu}\left(\xi_{n} x\right)\right\rangle .
$$

Then for each $\varphi(x) \in D(I), I=(0,1)$, we have

$$
\lim _{N \rightarrow \infty}\left\langle 2 \sqrt{x} \sum_{n=1}^{N} \frac{F\left(\xi_{n}\right)}{h_{n}} J_{\nu}\left(\xi_{n} x\right), \varphi(x)\right\rangle=\langle f(x), \varphi(x)\rangle .
$$

Third form :

$$
I=(a, b), 0<a<b<\infty, q(x)=\frac{\nu^{2}-\frac{1}{4}}{x^{2}}, \quad \nu \geq-\frac{1}{2} .
$$

The Sturm-Liouville problem is 


$$
\frac{d^{2} y}{d x^{2}}+\left(\lambda-\frac{\nu^{2}-\frac{1}{4}}{x^{2}}\right) y=0, \quad y(a)=y(b)=0
$$

In the present case

$$
\Psi_{n}(x)=\sqrt{\frac{\pi x}{2 h_{n}}}\left[J_{\nu}\left(\xi_{n} x\right) Y_{\nu}\left(\xi_{n} b\right)-J_{\nu}\left(\xi_{n} a\right) Y_{\nu}\left(\xi_{n} x\right)\right]
$$

where $Y_{\nu}(x)$ is the $\nu$ th order Bessel function of the second kind and $\xi_{n}$ are the positive roots of

$$
J_{\nu}(\xi a) Y_{\nu}(\xi b)-Y_{\nu}(\xi a) J_{\nu}(\xi b)=0
$$

with $0<\xi_{1}<\xi_{2}<\xi_{3}<\ldots$ Also,

$$
h_{n}=\xi_{n}^{-2}\left\{1-\left[\frac{J_{\nu}\left(\xi_{n} b\right)}{J_{\nu}\left(\xi_{n} a\right)}\right]^{2}\right\}
$$

$$
\lambda_{n}=\xi_{n}^{2} \text {. }
$$

As in the case of Hankel transform of the first form we can define the testing function $\mathscr{G}_{\nu}(I), I=(a, b)$ to be the collection of infinitely differentiable complex valued functions $\varphi(x)$ satisfying

$$
\rho_{k}(\phi)=\sup _{a<x<b}\left|\left(\frac{d^{2}}{d x^{2}}-q(x)\right)^{k} \phi(x)\right|<\infty, \quad k=0,1,2, \cdots
$$

The topology over $\mathscr{G}_{\nu}(I)$ is generated by the sequence $\left\{\rho_{k}\right\}_{k=0}^{\infty}$. The concept of convergence and completeness is defined in the usual way. Then the Sturm-Liouville expansion in the present case is given by

THEOREM 10. Let $f \in \mathscr{G}_{\nu}^{\prime}(I)$, where $I=(a, b), b>a>0$, and let $F\left(\xi_{n}\right)$ be the finite Hankel transform of the third form of $f$, where $\xi_{n}$ are the positive roots of (5.41), defined by

$$
F\left(\xi_{n}\right)=\left\langle f(x), \sqrt{x}\left[J_{\nu}\left(\xi_{n} x\right) Y_{\nu}\left(\xi_{n} b\right)-Y_{\nu}\left(\xi_{n} x\right) J_{\nu}\left(\xi_{n} a\right)\right]\right\rangle .
$$

Then for each $\varphi(x) \in D(I)$, we have

$$
\begin{aligned}
\lim _{N \rightarrow \infty}\left\langle\frac{\pi}{2} \sqrt{x} \sum_{n=1}^{N} \frac{F\left(\xi_{n}\right)}{h_{n}}\left[J_{\nu}\left(\xi_{n} x\right) Y_{\nu}\left(\xi_{n} b\right)-Y_{\nu}\left(\xi_{n} x\right) J_{\nu}\left(\xi_{n} a\right)\right], \varphi(x)\right\rangle \\
=\langle f(x), \varphi(x)\rangle .
\end{aligned}
$$


6. Expansion of generalized functions defined over a compact subset of $(a, b)$ when at least one of $a$ and $b$ is infinite

We will illustrate this case with $a=0$ and $b=\infty$ and the other cases can be similarly dealt with we will also assume that the eigenvalues associated with the corresponding Sturm-Liouville system are discrete. For the criterion of the discreteness of the eigenvalues of the Sturm-Liouville system one can see into [14; pp. 239-245] and [18, chapter VII]. The case when eigenfunctions are not discrete will be a subject of discussion in our next paper.

The following theorem due to Titchmarsh [18; p. 26] is the basis of the analysis presented in this section. For complete statement we reproduce the following terminology from [18; pp. 23-35].

Let $\phi(x)=\phi(x, \lambda), \theta(x)=\theta(x, \lambda)$ be the solutions of

$$
\frac{d^{2} y}{d x^{2}}-[\lambda-V(x)] y=0
$$

such that

$$
\begin{array}{ll}
\phi(0)=\sin \alpha, & \phi^{\prime}(0)=-\cos \alpha \\
\theta(0)=\cos \alpha, & \theta^{\prime}(0)=\sin \alpha
\end{array}
$$

where $\alpha$ is real.

If $m(\lambda)$ is the limit point, on any point on the limit circle, then for any non-real values $\lambda(6.0)$ has a solution

$$
\psi(x, \lambda)=\theta(x, \lambda)=\theta(x, \lambda)+m(\lambda) \phi(x, \lambda)
$$

belonging to $L^{2}(0, \infty)$. The functions denoted by $m(\lambda)$ in the upper and lower half planes are not necessarily analytic continuation of each other but we assume that they form a single analytic function whose only singularities are poles on the real axis. Let them be $\lambda_{0}, \lambda_{1}, \lambda_{2}, \ldots$ and let the corresponding residues be $\gamma_{0}, \gamma_{1}, \gamma_{2}, \cdots$. Then $\psi_{n}=\gamma_{n}^{1 / 2} \psi\left(x, \lambda_{n}\right)$ form a normal orthogonal set i.e.

$$
\int \psi_{n}^{2}(x) d x=1 \text { and } \int \psi_{n}(x) \psi_{m}(x) d x=0 \text { if } n \neq m .
$$

THEOREM 11 (Titchmarsh, 18; p. 26). Let $f(x)$ be the integral of an absolutely continuous function and let 


$$
\begin{gathered}
L_{x}\{f(x)\}=q(x) f(x)-f^{\prime \prime}(x) \text { be } L^{2}(0, \infty) ; \text { let } \\
f(0) \cos \alpha+f^{\prime}(0) \sin \alpha=0
\end{gathered}
$$

and

$\lim _{x \rightarrow \infty}\left\{\psi(x, \lambda) f^{\prime}(x)-\psi^{\prime}(x, \lambda) f(x)\right\}=0 \quad$ for every non-real $\lambda$. Then

$$
f(x)=\sum_{n=0}^{\infty} \psi_{n}(x) \int_{0}^{\infty} f(y) \psi_{n}(y) d y \quad(0 \leq x \leq \infty)
$$

the series being absolutely and uniformly convergent in any finite interval.

The testing function space $M(I)$. Let $K$ be an arbitrary compact subset of the interval $(0, \infty)$. The space $M(I)$ consists of infinitely differentiable complex valued functions $\varphi(x)$ satisfying

$$
\beta_{m, K}(\varphi) \triangleq \sup _{x \in K}\left|\Delta_{x}^{m} \varphi(x)\right|<\infty
$$

for each $m=0,1,2, \ldots$. The collection of seminorms $\left\{\beta_{m, K}\right\}$ generates the topology over $M(I)$. It turns out that $M(I)$ is a locally convex, sequentially complete Hausdorff topological vector space, the concept of convergence and completeness being defined in the usual way.

Now, we state and give an outline of the proof of the main expansion theorem.

THEOREM 12. Let $f(x) \in M^{\prime}(I)$ and $\Psi_{n}(x)$ be the normalized eigenfunctions of the Sturm-Liouville problem.

$$
\begin{gathered}
L_{x} \phi=\lambda \phi \\
\phi(0) \cos \alpha+\phi^{\prime}(0) \sin \alpha=0
\end{gathered}
$$

which are assumed to be elements of $M(I)$. Define the distributional Sturm-Liouville transform of $f$ by

$$
S[f] \triangleq F(n) \triangleq\left\langle f(x), \Psi_{n}(x)\right\rangle,
$$

$n=1,2,3, \cdots$ Then for each $\varphi(t) \in D(I)$,

$$
\lim _{N \rightarrow \infty}\left\langle\sum_{n=1}^{N} \Psi_{n}(t) F(n), \varphi(t)\right\rangle=\langle f(t), \varphi(t)\rangle
$$

Proof. Assume that the support of $\varphi(x)$ is contained in the finite 
interval $[c, d]$, where $0<c<d$. Then proceeding as in the proof of Theorem 2 we need to show that

$$
\int_{c}^{d} \sum_{n=1}^{N} \Psi_{n}(x) \Psi_{n}(t) \varphi(t) d t \rightarrow \varphi(x) \quad \text { in } M(I)
$$

uniformly for all $x$ as $N \rightarrow \infty$. That is, we have to show that

$$
\sum_{n=1}^{N} \Psi_{n}(x) \int_{0}^{\infty} \Psi_{n}(t) \varphi_{k}(t) d t \rightarrow \varphi_{k}(x)
$$

where $\varphi_{k}(t)=\Delta_{t}^{k} \varphi(t)$, for each $k=0,1,2, \ldots$. Since the function $\varphi_{k}(x)$ satisfies all the conditions of Theorem 11, the series in (6.6) converges uniformly and absolutely to $\phi_{k}(x)$ over $[c, d]$. This completes the proof of Theorem 12.

Remark. The case when $a=-\infty$ and $b=\infty$ can similarly be dealt with.

As a special case of Theorem 12 we obtain an expansion of the generalized function in a series of Laguerre polynomials [15; pp. 84, 87].

$$
I=(0, \infty), \quad q(x)=x^{2}+\frac{\alpha^{2}-\frac{1}{4}}{x^{2}}
$$

The Sturm-Liouville problem is

$$
\frac{d^{2} y}{d x^{2}}+\left(\lambda-x^{2}-\frac{\alpha^{2}-\frac{1}{4}}{x^{2}}\right) y=0
$$

where $y$ remains bounded as $x \rightarrow 0+$ and $x \rightarrow \infty$.

The eigenfunctions are

$$
\Psi_{n}(x)=\left(\frac{2 n !}{\Gamma(n+\alpha+1)}\right)^{1 / 2} x^{\alpha+\frac{1}{2}} e^{-\frac{1}{2} x^{2}} L_{n}^{\alpha}\left(x^{2}\right)
$$

and

$$
\lambda_{n}=4 n+2 \alpha+2, \quad n=0,1,2, \cdots .
$$

The testing function space $\mathscr{L}(I)$ consists of infinitely differentiable complex valued functions $\varphi(x)$ defined over an arbitrary compact subset $K$ of $I=(0, \infty)$ which satisfy

$$
\beta_{m, K}(\phi)=\sup _{x \in K}\left|\Delta_{x}^{m} \varphi(x)\right|<\infty,
$$


for each $m=0,1,2, \cdots$, where $\Delta_{x}=\frac{d^{2}}{d x^{2}}-x^{2}-\frac{\alpha^{2}-\frac{1}{4}}{x^{2}}$. The topology over $\mathscr{L}(I)$ is generated by $\left\{\beta_{m, K}\right\}_{m=0}^{\infty}$. The space $\mathscr{L}(I)$ possesses properties similar to that of $\mathscr{M}(I)$. The expansion theorem can be stated as

THEOREM 13. Let $f(x) \in \mathscr{L}^{\prime}(I)$ and $\Psi_{n}(x)$ be the normalized eigenfunctions of the Sturm-Liouville problem (6.8). Define the distributional Laguerre transform of $f$ by

$$
F(n) \triangleq\left\langle f(x), x^{\alpha+\frac{1}{2}} e^{-\frac{1}{2} x 2} L_{n}^{\alpha}\left(x^{2}\right)\right\rangle .
$$

Then for each $\varphi \in D(I)$,

$$
\lim _{N \rightarrow \infty}\left\langle\sum_{n=1}^{N} F_{n} \frac{2 n !}{\Gamma(n+\alpha+1)} t^{\alpha+\frac{1}{2}} e^{-\frac{1}{2} t^{2}} L_{n}^{\alpha}\left(t^{2}\right), \varphi(t)\right\rangle=\langle f(t), \varphi(t)\rangle .
$$

\section{An operational calculus}

The Sturm-Liouville transform is useful in solving a class of boundary value problems.

Let us define an operator $\Delta_{x}^{*}: \mathscr{N}^{\prime}(I) \nrightarrow \mathscr{N}^{\prime}(I)$ by the relation

$$
\left\langle\Delta_{x}^{*} f(x), \varphi(x)\right\rangle \triangleq\left\langle f(x), \Delta_{x} \varphi(x)\right\rangle
$$

for all $f \in \mathscr{N}^{\prime}(I)$ and $\varphi(x) \in \mathscr{N}(I), I=(a, b)$. It is a simple exercise to show that for $\varphi(x) \in \mathscr{N}(I)$,

$$
\left\langle\left(\Delta_{x}^{*}\right)^{k} f(x), \varphi(x)\right\rangle=\left\langle f(x), \Delta_{x}^{k} \varphi(x)\right\rangle, \quad k=1,2,3, \cdots .
$$

It is a fact that if $f$ is a regular distribution in $\mathscr{N}^{\prime}(I)$ generated by a member of $D(I)$ then

$$
\Delta_{x}^{*} f \equiv \Delta_{x} f
$$

It readily follows that

$$
\left\langle\left(\Delta_{x}^{*}\right)^{k} f(x), \psi\left(\lambda_{n}, x\right)\right\rangle=\left(-\lambda_{n}\right)^{k}\left\langle f(x), \Psi\left(\lambda_{n}, x\right)\right\rangle
$$

where $\Psi\left(\lambda_{n}, x\right)$ stands for the usual eigenfunction $\Psi_{n}(x)$ corresponding to the eigenvalue $\lambda_{n}$.

Or, in otherwords

$$
S\left[\left(\Delta_{x}^{*}\right)^{k} f(x)\right]=\left(-\lambda_{n}\right)^{k} S[f(x)], \quad \text { for each } k=1,2,3, \cdots
$$


We can use this fact to solve the general operator equation

$$
P\left(\Delta_{x}^{*}\right) u=g
$$

where $P$ is a polynomial, the given $g$ and the unknown $u$ are required to be in $\mathscr{N}^{\prime}(I)$.

Applying the Sturm-Liouville transform to (7.3) we obtain

$$
P\left(-\lambda_{n}\right) U\left(\lambda_{n}\right)=G\left(\lambda_{n}\right)
$$

where $U$ and $G$ are the Sturm-Liouville transforms of $u$ and $g$ respectively. If $P\left(-\lambda_{n}\right) \neq 0$, for every $n$, we can divide by $P\left(-\lambda_{n}\right)$ and apply the inverse transform to get

$$
u(x)=\sum_{n=0}^{\infty} \frac{G\left(\lambda_{n}\right)}{P\left(-\lambda_{n}\right)} \Psi_{n}(x) .
$$

If $P\left(-\lambda_{n}\right)=0$ for some $\lambda_{n}$, say, $\lambda_{n_{k}}(k=1, \cdots, m)$, then the solution exists in $\mathscr{N}^{\prime}$ if and only if $G\left(\lambda_{n_{k}}\right)=0$, for $k=1, \cdots, m$. In this case a solution to (7.3) is

$$
u(x)=\sum_{P\left(-\lambda_{n}\right) \neq 0} \frac{G\left(\lambda_{n}\right)}{P\left(-\lambda_{n}\right)} \Psi_{n}(x)+\sum_{k=1}^{m} a_{k} \Psi_{n_{k}}(x),
$$

where the $a_{k}$ are arbitrary constants [24, p. 265].

In a given boundary value problem it is easy to verify that (7.5) and (7.6) satisfy the given differential equation and the prescribed boundary conditions as is shown in the following sections 8 and 9 .

8. Dirichlet problem for the interior of a unit sphere (Application of the Legendre transformation)

Find the conventional function $u(r, x)$ satisfying the differential equation

$$
\frac{\partial}{\partial x}\left[\left(1-x^{2}\right) \frac{\partial u}{\partial x}\right]+r \frac{\partial^{2}}{\partial r^{2}}(r u)=0 \quad(0<r<1,-1<x<1)
$$

such that

(i) $u(r, x) \rightarrow f(x)$ in $\mathscr{P}^{\prime}(I), I=(-1,1)$, as $r \rightarrow 1-$,

(ii) $u(r, x)$ remains bounded as $r \rightarrow 0+$

and 
(iii) $\left(1-x^{2}\right) u(r, x),\left(1-x^{2}\right) \frac{\partial u}{\partial x}(r, x) \rightarrow 0$ as $x \rightarrow \pm 1$.

Now, we proceed to solve our main problem. Applying Legendre transformation to (8.1) and denoting the Legendre transformation of $u(r, x)$ by $\bar{u}(r, n)$ we have

$$
-n(n+1) \bar{u}+r \frac{\partial^{2}}{\partial r^{2}}(r \bar{u})=0
$$

The solution of this Euler equation is

$$
\bar{u}(r, n)=A(n) r^{n}+B(n) r^{-n-1} .
$$

Since $u(r, x)$ remains finite in a neighbourhood of the zero we set $B(n)$ $=0$. So that

$$
\bar{u}(r, n)=A(n) r^{n}
$$

Also, as $r \rightarrow 1-, u(r, x) \rightarrow f(x)$, therefore we formally write

$$
A(n)=F(n)=\left\langle f(x), P_{n}(x)\right\rangle .
$$

So that

$$
\bar{u}(r, n)=r^{n}\left\langle f(x), P_{n}(x)\right\rangle .
$$

Now, applying the inversion formula (5.22) for the Legendre transformation we get

$$
u(r, x)=\lim _{N \rightarrow \infty} \sum_{n=0}^{N}\left(n+\frac{1}{2}\right) P_{n}(x) r^{n}\left\langle f(t), P_{n}(t)\right\rangle
$$

in $D^{\prime}(I), I=(-1,1)$.

To verify that this is indeed the solution of our problem we proceed as follows: By the boundedness property of generalized functions there exist a positive constant $C$ and a nonnegative integer $q$ such that

$$
\begin{aligned}
\left|\left\langle f(t), P_{n}(t)\right\rangle\right| & \leq C \max _{0 \leq k \leq q} \sup _{-1<t<1}\left|\nabla_{t}^{k}\left(\left(1-t^{2}\right)^{1 / 2} P_{n}(t)\right)\right| \\
& \leq C \max _{0 \leq k \leq q} \sup _{-1<t<1}\left|n^{k}(n+1)^{k}\left(1-t^{2}\right)^{1 / 2} P_{n}(t)\right| \\
& \leq C n^{q}(n+1)^{q} .
\end{aligned}
$$

Therefore, 


$$
\begin{aligned}
\sum_{n=0}^{N}(n+ & \left.\frac{1}{2}\right) P_{n}(x) r^{n}\left\langle f(t), P_{n}(t)\right\rangle \\
& \leq C \sum_{n=0}^{N} r^{n}[n(n+1)]^{q}\left(n+\frac{1}{2}\right) .
\end{aligned}
$$

Since the series on the right converges for $0<r \leqslant r_{0}<1$, it follows that the series on the left-hand side is uniformly convergent. The same result can be shown to be still true if we differentiate the left-hand side with respect to $x$ (also with respect to $r$ ) any number of times and use the formula

$$
\left|P_{n}^{(k)}(x)\right| \leq n^{2 k}
$$

for each $k=1,2,3, \cdots$ and $|x|<1$. Therefore, we can apply the operator $\frac{\partial}{\partial x}\left[\left(1-x^{2}\right) \frac{\partial}{\partial x}\right]+r\left(\frac{\partial^{2}}{\partial r^{2}}\right) r$ to the series (8.4) term by term and see that $u(r, x)$ satisfies in the conventional sense the differential equation (8.1).

Next, by virtue of the uniform convergence of the series (8.4) we can take the limit $r \rightarrow 0+$ in (8.4) and see that it converges to $\frac{1}{2}\langle f(t)$, $P_{0}(t)>$. This verifies (ii).

Finally we verify the first boundary condition. Let $Q(x)$ be a polynomial of degree $q+2$ having no zeros on the positive real axis and let $\Delta_{x}$ denote the operator $\frac{\partial}{\partial x}\left[\left(x^{2}-1\right) \frac{\partial}{\partial x}\right]$. Then, for any $\varphi \in D(I)$ with its support contained in $[c, d]$ where $-1<c<d<1$, we have

$$
\begin{aligned}
\langle u(r, x), \varphi(x)\rangle & =\lim _{N \rightarrow \infty}\left\langle\sum_{n=0}^{N}\left(n+\frac{1}{2}\right) P_{n}(x) r^{n} F(n), \varphi(x)\right\rangle \\
& =\int_{c}^{d} \sum_{n=0}^{N}\left(n+\frac{1}{2}\right) r^{n} F(n) P_{n}(x) \varphi(x) d x .
\end{aligned}
$$

As the series remains uniformly convergent after applications of $\Delta_{x}$ to it any number of times for $r<1$, we can write

$$
\begin{aligned}
&\langle u(r, x), \varphi(x)\rangle=\lim _{N \rightarrow \infty} \sum_{n=0}^{N}\left(n+\frac{1}{2}\right) \frac{r^{n} F(n)}{Q[n(n+1)]} \int_{c}^{d} Q\left(\Delta_{x}\right)\left[P_{n}(x)\right] \varphi(x) d x \\
&=\lim _{N \rightarrow \infty} \sum_{n=0}^{N}\left(n+\frac{1}{2}\right) \frac{r^{n} F(n)}{Q[n(n+1)]} \int_{c}^{d} P_{n}(x) Q\left(\Delta_{x}\right) \varphi(x) d x \\
& \text { (integrating by parts) }
\end{aligned}
$$


Therefore,

$$
|\langle u(r, x), \varphi(x)\rangle| \leqslant \lim _{N \rightarrow \infty} \sum_{n=0}^{N} \frac{\left(n+\frac{1}{2}\right) r^{n} F(n)}{Q[n(n+1)]} \int_{c}^{d}\left|Q\left(\Delta_{x}\right) \varphi(x)\right| d x
$$

using the fact that $\left|P_{n}(x)\right| \leqslant 1$ for $-1 \leq x \leq 1$. Now, we see that the series on the right-hand side is uniformly convergent for $0<r \leq 1$. Hence, we can take the limit $r \rightarrow 1-$ in (8.8) and conclude that

$$
\lim _{r \rightarrow 1-}\langle u(r, x), \varphi(x)\rangle=\sum_{n=0}^{\infty}\left\langle\left(n+\frac{1}{2}\right) F(n) P_{n}(x), \varphi(x)\right\rangle=\langle f, \varphi\rangle .
$$

In view of the inequalities (8.5) and (8.7) the series $\sum_{n=0}^{\infty}\left(n+\frac{1}{2}\right) P_{n}^{\prime}(x) r^{n}$ $\left\langle f(t), P_{n}(t)\right\rangle$ converges uniformly in $(-1,1)$ for a fixed $r$ satisfying $0<r<1$.

Therefore, $\frac{\partial u}{\partial x}=\sum_{n=0}^{\infty}\left(n+\frac{1}{2}\right) P_{n}^{\prime}(x) r^{n}\left\langle f(t), P_{n}(t)\right\rangle$ and is bounded uniformly for all $x$ in $(-1,1)$ and a fixed $r$ in $(0,1)$. This verifies (iii).

9. Temperature in a long cylinder (Application of the finite Hankel transform of the first form)

The problem can be stated as below:

Find the conventional function $u(r, t)$ on the domain

$$
\{(r, t): 0<r<1, t>0\}
$$

satisfying the heat equation

$$
\frac{\partial u}{\partial t}=K\left(\frac{\partial^{2} u}{\partial r^{2}}+\frac{1}{r} \frac{\partial u}{\partial r}\right)
$$

where $K$ is a positive constant, and the distributional boundary conditions :

(i) As $t \rightarrow 0+, u(r, t)$ converges in $D^{\prime}(I), I=(0,1)$ to a certain generalized function $f(r) \in \mathscr{H}_{0}^{\prime}(I)$.

(ii) As $r \rightarrow 1-0, u(r, t)$ converges uniformly to zero for each fixed $t>0$.

Using the change of variables

$$
v(r, t)=\sqrt{r} u(r, t), \quad g(r)=\sqrt{r} f(r)
$$

the equation assumes the form 


$$
\frac{\partial v}{\partial t}=K\left(\frac{\partial^{2} v}{\partial r^{2}}+\frac{1}{4} \frac{v}{r^{2}}\right)
$$

to which our zero order finite Hankel transform can be applied. Denoting the zero order finite Hankel transform of $v(r, t)$ with respect to $r$ by $V\left(\xi_{i}, t\right)$, where $\xi_{i}$ are the positive roots of $J_{0}(\xi)=0$, an application of the transform converts (9.3) into

$$
\frac{\partial V}{\partial t}=-\frac{\xi_{i}^{2}}{K} V
$$

So that

$$
V\left(\xi_{i}, t\right)=A\left(\xi_{i}\right) e^{-\left(\xi_{i}^{2} / K\right) t} .
$$

In view of the boundary condition (i) and (9.3)

$$
A\left(\xi_{i}\right)=\left\langle g(x), \sqrt{x} J_{0}\left(x \xi_{i}\right)\right\rangle .
$$

Notice that $g(r) \in \mathscr{H}_{0}^{\prime}(I)$ because $f(r) \in \mathscr{H}_{0}^{\prime}(I)$. Hence,

$$
V\left(\xi_{i}, t\right)=e^{-\left(\xi_{i}^{2} / K\right) t}\left\langle g(x), \sqrt{x} J_{0}\left(x \xi_{i}\right)\right\rangle .
$$

Formally taking inverse transform (5.29) we have

$$
u(r, t)=\lim _{N \rightarrow \infty} 2 \sqrt{r} \sum_{i=1}^{\infty} \frac{e^{-\left(\xi_{i}^{2} / K\right) t}}{J_{1}^{2}\left(\xi_{i}\right)}\left\langle g(x), \sqrt{x} J_{0}\left(x \xi_{i}\right)\right\rangle J_{0}\left(r \xi_{i}\right)
$$

in $D^{\prime}(I)$.

We now verify that (9.4) is truly a solution. By the boundedness property of generalized functions there exist a positive constant $C$ and a nonnegative integer $s$ such that

$$
\begin{aligned}
\left|A\left(\xi_{i}\right)\right| & =\left|\left\langle g(x), \sqrt{x} J_{0}\left(x \xi_{i}\right)\right\rangle\right| \leq C \max _{0 \leqslant k \leqslant s} \gamma_{k}\left(\sqrt{x} J_{0}\left(x \xi_{i}\right)\right) \\
& =C \max _{0 \leq k \leq s} \sup _{0<x<1}\left|\left(\frac{d^{2}}{d x^{2}}+\frac{1}{4 x^{2}}\right)^{k} \sqrt{x} J_{0}\left(x \xi_{i}\right)\right| \\
& =C \max _{0 \leq k \leq s} \sup _{0<x<1}\left|x^{\frac{1}{2}} \xi_{i}^{2 k} J_{0}\left(x \xi_{i}\right)\right| \\
& \leq C^{\prime} \xi_{i}^{2 s} .
\end{aligned}
$$

for some appropriate constant $C^{\prime}$. Therefore, in view of the results [10, pp. 147 and 153]

$$
\xi_{i} \sim \pi\left(i-\frac{1}{4}\right) \quad i \rightarrow \infty
$$


and

$$
\left|J_{1}\left(\xi_{i}\right)\right|^{-1} \leq R \sqrt{i} \quad i=1,2,3, \cdots, R>0
$$

we have

$$
\begin{gathered}
\sum_{i=1}^{N} \frac{e^{-\left(\xi_{i}^{2} / K\right) t}}{J_{1}^{2}\left(\xi_{i}\right)}\left|A\left(\xi_{i}\right)\right| J_{0}\left(r \xi_{i}\right) \\
\leq C^{\prime} K \sum_{i=1}^{N}\left|e^{-\left(\xi_{i}^{2} / K\right) t_{i}} \xi_{i}^{2 s}\right| \leq C^{\prime \prime} \sum_{i=1}^{N} e^{-\pi^{2} i^{2} t / K} i^{2 s+1} .
\end{gathered}
$$

Clearly the series on the right is always convergent for $t>0$. Hence the series (9.4) converges uniformly and absolutely for all $t>0$ and $0<r<1$. Since $J_{0}\left(r \xi_{i}\right)$ and $J_{1}\left(r \xi_{i}\right)$ are uniformly bounded for $0<r<1$ and $i=1,2,3, \cdots$, the same conclusion remains true when the operators $\frac{\partial^{2}}{\partial r^{2}}, \frac{1}{r} \frac{\partial}{\partial r}$ and $\frac{\partial}{\partial t}$ respectively are applied to (9.4). In fact application of the operator $K\left(\frac{\partial^{2}}{\partial r^{2}}+\frac{1}{4 r^{2}}\right)-\frac{\partial}{\partial t}$ to (9.4) yields result zero. In this way we verify that (9.4) satisfies the differential equation (9.3).

Since the series (9.4) is uniformly convergent for $t>0$ we can take the limit $r \rightarrow 1-$ within the summation sign in (9.4). As $J_{0}\left(\xi_{i}\right)=0$, obviously $r \rightarrow 1-$ implies $v(r, t)$ uniformly for all $t>0$.

Finally, we consider the case $t \rightarrow 0+$. In fact for any $\varphi(r) \in D(I)$, $I=(c, d)$, we have

$$
\begin{aligned}
|\langle v(r, t), \varphi(r)\rangle| & =\left|\lim _{N \rightarrow \infty}\left\langle 2 r \sum_{i=0}^{N} \frac{e^{-\xi_{i}^{2} t / K}}{J_{1}^{2}\left(\xi_{i}\right)} A\left(\xi_{i}\right) J_{0}\left(r \xi_{i}\right), \varphi(r)\right\rangle\right| \\
& =\lim _{N \rightarrow \infty} 2 \sum_{i=0}^{N} \frac{e^{-\xi_{i}^{2} t / K}}{J_{1}^{2}\left(\xi_{i}\right)}\left|A\left(\xi_{i}\right)\right| \int_{c}^{d} r J_{0}\left(r \xi_{i}\right) \varphi(r) d r \\
& \leq \lim _{N \rightarrow \infty} 2 \sum_{i=0}^{N} C e^{-\xi_{i}^{2} t / K} i \xi_{i}^{2 s}\left|\int_{c}^{d} r J_{0}\left(r \xi_{i}\right) \varphi(r) d r\right|
\end{aligned}
$$

for a positive constant $C$ and a non-negative integer $s$ [see 24, p. 19]. Now, integrating by parts the last integral and using the facts that

$$
\begin{gathered}
\int J_{1}(x) d x=J_{0}(x), \quad \int x J_{0}(x) d x=x J_{1}(x), \\
\varphi^{(k)}(c)=\varphi^{(k)}(d)=0 \quad \text { for all } k=0,1,2, \cdots,
\end{gathered}
$$

we can see that 


$$
\mid\left\langle v(r, t), \varphi(t)\left|\leqslant 2 C \lim _{N \rightarrow \infty} \sum_{i=0}^{N} e^{-\xi_{i}^{2} t / K} i \frac{\xi_{i}^{2 s}}{\xi_{i}^{2 s+3}}\right| \int_{c}^{d}\left(\sum_{j=i}^{2 s+3} r P_{j}\left(\frac{1}{r}\right) \varphi^{(j)}(r)\right) J_{1}\left(r \xi_{i}\right) d r\right|
$$

where $P_{j}\left(\frac{1}{r}\right)$ is a certain polynomial in $1 / r$. Thus

$$
\begin{aligned}
\langle v(r, t), \varphi(t)\rangle & \leq 2 C \lim _{N \rightarrow \infty} \sum_{i=0}^{N} \frac{e^{-\xi_{i}^{2} t / K} i}{\xi_{i}^{3}} \int_{c}^{a}\left|\sum_{j=1}^{2 s+1} r P_{j}\left(\frac{1}{r}\right) \varphi^{(j)}(r)\right| d r \\
& <C^{\prime} \sum_{i=0}^{N} \frac{1}{i^{2}} \quad(\text { by (9.6)) }
\end{aligned}
$$

where $C^{\prime}$ is a certain constant. Since the series on the right of (9.11) is convergent independently of $t$, we can take the limit $t \rightarrow 0+$ in (9.10) and arrive at

$$
\lim _{t \rightarrow 0+}\langle v(r, t), \varphi(r)\rangle=\lim _{N \rightarrow \infty}\left\langle 2 r \sum_{i=0}^{N} \frac{F\left(\xi_{i}\right)}{J_{1}^{2}\left(\xi_{i}\right)} J_{0}\left(r \xi_{i}\right), \varphi(r)\right\rangle,
$$

verifying thereby the second boundary condition.

This work was supported by National Research Council grant no. A5298 and Centre de Recherches Mathématiques, Université de Montréal, P. Q., Canada.

\section{REFERENCES}

[ 1 ] Bouix, M., Les fonctions généralisees ou distributions, Masson, Paris, 1964.

[ 2 ] Braga, C. L. R. and M. Schönberg, Formal series and distributions. An de Acad. Brasileira de Ciências, 31 (1959), 333-360.

[ 3 ] Churchill, R. V., Fourier series and boundary value problems, McGraw-Hill, New York, 1963.

[4] Dube, L. S., Finite Hankel transformation of a class of generalized functions, Pacific J. Math., 62(2) (1976), 365-378.

[ 5] Erdélyi, A. (Editor), Higher Transcendental functions, Vol. 11 McGraw-Hill, New York, 1953.

[6] Eringen, A. C., The finite Sturm-Liouville transform, Quart. J. Math. Oxford, (2), 5 (1954), 120-129.

[ 7 ] Gelfand, I. M. and G. E. Shilov, Generalized functions, vols. 1 and 3 Academic Press, New York, 1964, 1967.

[8] Giertz, M., On the expansion of certain generalized functions in series of orthogonal functions, Proc. London Math. Soc., 3rd ser., 14 (1964), 45-52.

[ 9 ] Ince, E. L., Ordinary differential equations, Dover publications, New York, 1926.

[10] Jahnke, E., F. Emde and F. Losch, Tables of higher functions, McGraw-Hill, New York, 1960.

[11] Korevaar, J., Pansions and the theory of Fourier Transforms, Trans. Amer. Math. Soc., 91 (1959), 53-101.

[12] - Distributions defined from the point of view of applied mathematics, Kon. Ned. Akad. Wetensch. Proc. Ser. A, 58 (1955), 368-389, 483-503, 663-674. 
[13] Lighthill, M. J., Fourier analysis and generalized functions, Cambridge Univ. Press, 1958.

[14] Naimark, M. A., Linear differential operators part II, Frederick Ungar Publishing Co., New York, 1968.

[15] Schwartz, L., Theore des Distributions, Vol. I, II, Hermann, Paris 1957, 1959.

[16] Sneddon, I. N., The use of integral transforms, McGraw-Hill, New York, 1972.

[17] Temple, G., The theory of generalized functions, Proc. Roy. Soc., Ser. A, 228 (1955), 175-190.

[18] Titchmarsh, E. C., Eigenfunction expansions associated with second order differential equations, Vol 1, Clarendon Press, Oxford, 1946.

[19] Walter, G. G., Expansions of distributions, Trans. Amer. Math. Soc., 116 (1965), 492-510.

[20] Widlund, O., On the expansion of generalized functions in series of Hermite functions, Kgl. Tekn. Hogsk. Mandl. Stockholm, No. 173, 1961.

[21] Warmbrod, G. K., The distributional finite Fourier transform, SIAM J. Appl. Math., 17 (5), (1969), 930-956.

[22] Yosida, K., Lectures on differential and integral equations, Interscience Publishers, New York, 1960.

[23] Zemanian, A. H., Orthonormal series expansions of certain distributions and distributional transform calculus, J. Math. Analysis and App. 14, No. 2 (1966), 263-275.

[24] —-, Generalized integral transformations, Interscience Publishers, New York, 1968.

Centre de Recherches Mathématiques, Université de Montréal

and

Department of Mathematics, Carleton University 
